\title{
SOLAR GAMMA RAYS AND THEIR CORRELATION WITH SPACE AND GROUND-BASED OBSERVATIONS
}

\author{
G. G. FAZIO \\ (Smithsonian Astrophysical Observatory and Harvard College Observatory, \\ Cambridge, Mass., U.S.A.)
}

Thus far, only two experiments have detected solar $\gamma$-radiation with energy significantly greater than $200 \mathrm{keV}$. In both events the $\gamma$-ray emission occurred during a solar flare. The first observation was in 1958 by Peterson and Winckler (1959), who recorded a burst of radiation that occurred in less than $18 \mathrm{sec}$ from a class-2 solar flare. The radiation spectrum peaked in the 200- to 500-keV region. Recently, Cline et al. (1967) recorded in the OGO-3 satellite three rapid $\gamma$-ray bursts in the $80-\mathrm{keV}$ to $1-\mathrm{MeV}$ energy range and measured the integral energy spectrum. The measurements were made on July 7, 1966, during the first high-intensity flare (importance 3 ) of the new solar cycle. Many attempts have been made to measure higher energy $\gamma$-radiation from the quiet Sun and from solar flares, but no flux has been detected; this is primarily due to the fact that no high-energy $\gamma$-ray detectors have viewed a major solar flare during the maximum of the optical or microwave burst. However, theoretical estimates of the flux of solar $\gamma$-rays, based on a simple flare model, indicate a readily detectable flux from a major flare even to photon energies of $100 \mathrm{MeV}$. It is therefore important that experiments be performed during the coming maximum of the solar cycle to investigate this region of the electromagnetic spectrum.

To understand better how solar $\gamma$-ray measurements can improve our knowledge of flares and to specify what other simultaneous observations should be performed, let us briefly review the origin of $\gamma$-rays in a flare region. Gamma radiation from the Sun results primarily from the interactions of high-energy electrons, protons, and nuclei in the solar atmosphere. The intensity of the radiation is proportional to the product of the high-energy particle intensity and the solar gas density in the volume where the particles interact. The duration of the prompt $\gamma$-ray burst is related to the length of time the particles are trapped. The important source mechanisms are:

(1) Electron bremsstrahlung.

(2) Compton scattering (inverse Compton effect).

(3) Nuclear deexcitation.

(4) Positron-electron annihilation.

(5) Decay of $\pi^{0}$ mesons.

From theoretical calculations the most important source mechanism in the $100-\mathrm{keV}$ to $1-\mathrm{MeV}$ energy region is bremsstrahlung by high-energy electrons. The photon ener-

Perek (ed.), Highlights of Astronomy, 544-546. (C) I.A.U. 
gy spectrum is continuous. Line emission occurs at $0.51 \mathrm{MeV}$ because of positron annihilation, at $2.23 \mathrm{MeV}$ because of deuteron formation, and throughout the spectrum from 0.5 to $10 \mathrm{MeV}$ because of nuclear deexcitation. The most important lines in the last energy band are at $4.43 \mathrm{MeV}$ owing to $\mathrm{C}^{14}$ and at $6.14 \mathrm{MeV}$ owing to $\mathrm{O}^{16}$. All the above sources of line emission indicate the occurrence of nuclear reactions during a flare. The intensity of the $2 \cdot 23-\mathrm{MeV}$ line is a measure of the neutron density in the flare region. The $\gamma$-ray flux above $20 \mathrm{MeV}$ is primarily caused by $\pi^{0}$-meson decay, which produces a continuous energy spectrum peaking at about $70 \mathrm{MeV}$. The $\pi^{0}$ mesons are produced in high-energy proton and nucleon collisions with the solar gas.

The most important $\gamma$-ray measurements that can be performed are the following:

(1) The flux and energy spectrum as a function of time of the electron bremsstrahlung continuum. Microwave bursts that accompany X-ray radiation and $\gamma$-radiation may be caused by the synchrotron radiation of the same electrons.

(2) Time dependence of the flux of $\gamma$-ray line emission at $4.43 \mathrm{MeV}$ and $6 \cdot 14 \mathrm{MeV}$. This radiation is prompt and accurately reflects the time dependence of the product of the high-energy proton intensity and gas density in the flare volume. The $0 \cdot 51-\mathrm{MeV}$ and $2 \cdot 23-\mathrm{MeV}$ lines are delayed from the primary interactions and are therefore less useful. In addition, the $0.51-\mathrm{MeV}$ line may be completely masked by the bremsstrahlung radiation.

(3) The flux and energy spectrum of the radiation above $20 \mathrm{MeV}$. This radiation is also prompt and accurately reflects the time dependence of the product of the highenergy proton intensity and gas density in the flare volume. In addition, the intensity of the radiation is very sensitive to the energy of the accelerated protons.

From the nature of the source mechanisms it is evident that knowledge of the time dependence of the solar $\gamma$-ray energy spectrum and flux can yield direct and important new information on the manner in which high-energy electrons, protons, and nuclei in a flare are accelerated. Gamma radiation is not affected by solar and interplanetary magnetic fields and is not readily absorbed; therefore, flux and energy measurements are directly related to the source mechanism. However, the value of $\gamma$-ray experiments in interpreting acceleration mechanisms is dependent on other simultaneous measurements from both satellites and ground-based observatories.

Probably the most important supporting observations are in the X-ray and radio regions of the spectrum. Time correlations of the fine structure of radio, X-ray, and $\gamma$-ray solar bursts are essential for the understanding of the motions of high-energy electrons. In the radio spectrum, microwave bursts appear most directly related to high-energy X-ray and $\gamma$-ray emission. Location of the fine space structure of X-ray radiation and radio radiation on the solar disk is extremely desirable. Heliographs of high spatial resolution $\left(10^{\prime \prime}\right)$ at $\mathrm{X}$-ray and radio wavelengths have already been proposed by De Jager (1964).

The production of secondary nuclear particles such as neutrons, deutrons, tritons, 
and $\mathrm{He}^{3}$ is proportional to the product of the mean gas density and the length of time the high-energy particles are trapped. It is therefore important to measure the flux and energy of these particles that have escaped into the interplanetary medium, and to combine these data with $\gamma$-ray measurements to understand the details of the particleacceleration mechanism. The high-energy neutron measurements are of particular value since these particles are not affected by magnetic fields.

The flux and energy spectrum of high-energy electrons, protons, and $\alpha$ particles injected into the interplanetary medium during a solar flare can also be correlated with $\gamma$-ray measurements to understand the trapping and modulation of these particles.

Finally, knowledge of the magnetic-field structure in the solar-flare region, particularly before and after a flare, is necessary for the understanding of the particular acceleration process.

It may be premature to discuss what observations should be correlated with solar $\gamma$-ray bursts when so few bursts have been detected, but the importance of these observations cannot be overemphasized. More experiments should be performed during the coming maximum of the solar cycle to measure solar $\gamma$-rays during a flare. At energies above $1 \mathrm{MeV}$, very few experiments are currently planned for satellites.

\section{References}

Cline, T.L., Holt, S.S., Hones, E.W., Jr. (1967) NASA Preprint X-611-67-348.

De Jager, C. (1964) Research in Geophysics, Vol. I, The Massachusetts Institute of Technology Press, Cambridge, Mass., p. 1.

Peterson, L.E., Winckler, J.R. (1959) J. geophys. Res., 64, 697. 